\title{
Effects of Metformin on Hepatic Steatosis in Adults with Nonalcoholic Fatty Liver Disease and Diabetes: Insights from the Cellular to Patient Levels
}

\author{
Kanokwan Pinyopornpanish ${ }^{1}$, Apinya Leerapun ${ }^{1}$, Kanokporn Pinyopornpanish ${ }^{2}$, and Nipon Chattipakorn $3,4,5$ \\ ${ }^{1}$ Division of Gastroenterology, Department of Internal Medicine, ${ }^{2}$ Department of Family Medicine, ${ }^{3}$ Cardiac Electrophysiology Research \\ and Training Center, and ${ }^{4}$ Cardiac Electrophysiology Unit, Department of Physiology, Faculty of Medicine, Chiang Mai University, and \\ ${ }^{5}$ Center of Excellence in Cardiac Electrophysiology Research, Chiang Mai University, Chiang Mai, Thailand
}

\section{Article Info}

Received December 16, 2020

Revised February 16, 2021

Accepted February 17, 2021

\section{Corresponding Author}

Nipon Chattipakorn

ORCID https://orcid.org/0000-0003-3026-718X

E-mail nchattip@gmail.com

\begin{abstract}
Nonalcoholic fatty liver disease (NAFLD) patients with diabetes constitute a subgroup of patients with a high rate of liver-related complications. Currently, there are no specific drug recommendations for these patients. Metformin, a conventional insulin sensitizer agent, has been widely prescribed in patients with diabetes. Metformin treatment has been shown to be effective at alleviating hepatic lipogenesis in animal models of NAFLD, with a variety of mechanisms being deemed responsible. To date, most studies have enrolled diabetic patients who are treated with metformin, with the drug being taken continuously throughout the study. Although evidence exists regarding the benefits of metformin for NAFLD in preclinical studies, reports on the efficacy of metformin in adult NAFLD patients have had some discrepancies regarding changes in liver biochemistry and hepatic fat content. Evidence has also suggested possible effects of metformin as regards the prevention of hepatocellular carcinoma tumorigenesis. This review was performed to comprehensively summarize the available in vitro, in vivo and clinical studies regarding the effects of metformin on liver steatosis for the treatment of adult NAFLD patients with diabetes. Consistent reports as well as controversial findings are included in this review, and the mechanistic insights are also provided. In addition, this review focuses on the efficacy of metformin as a monotherapy and as a combined therapy with other antidiabetic medications. (Gut Liver 2021;15:827-840)
\end{abstract}

Key Words: Diabetes mellitus; Non-alcoholic fatty liver disease; Non-alcoholic steatohepatitis

\section{INTRODUCTION}

Nonalcoholic fatty liver disease (NAFLD) is a common disease with increasing incidence worldwide. A large cohort in the United States showed a 5-fold increase in incidence from 1997 to $2014 .{ }^{1}$ Insulin resistance has a pivotal role in NAFLD development and progression ${ }^{2}$ and NAFLD patients with diabetes are a subgroup of patients with a high rate of liver-related complications. ${ }^{3}$ In response to insulin resistance, hyperinsulinemia occurs causing the augmentation of hepatic de novo lipogenesis pathways, resulting in hepatic steatosis and further hepatic inflammation. ${ }^{4}$ Currently, the treatment of NAFLD is markedly under investigation. To date, no medication has been approved to treat NAFLD and nonalcoholic steatohepatitis (NASH) by the Food and Drug Administration in the United States and there is no specific drug recommended for treating the subgroup of NAFLD patients with diabetes.

Metformin, an insulin sensitizer agent in the biguanide subclass, is a widely used drug in diabetic patients with a good safety profile. Since it involves multiple molecular mechanisms in glucose metabolism and anti-inflammatory effects, ${ }^{5}$ metformin is one of the most interesting medications for the possible treatment or control of NAFLD progression. A previous meta-analysis of randomized-controlled trials evaluated the treatment response of metformin in patients with NAFLD and NASH. ${ }^{6}$ It was concluded that metformin was not associated with liver histologic 
improvement in patients with histologic NASH. However, most of the patients enrolled in these studies were nondiabetic ${ }^{7-11}$ or patients with insulin resistance without established diabetes. ${ }^{12}$ Currently, reports on the effects of metformin among diabetic NAFLD patients have been inconsistent. Since most of the diabetes patients were receiving metformin, the beneficial effects of this treatment need to be elucidated.

In this review, we have comprehensively summarized findings from in vivo, in vitro, and clinical studies regarding metformin for the treatment of adult NAFLD patients with diabetes. Our review focuses on the efficacy of metformin in treating liver steatosis. Consistent and controversial reports regarding the mechanisms responsible for the effect of metformin on NAFLD development are also discussed. Relevant publications in the PubMed database were included in this review, the search terms used being "metformin" and "NAFLD," "NASH" and "diabetes." Only the articles published in English were reviewed.

\section{EFFECTS OF METFORMIN ON LIPOGENESIS REDUCTION IN NAFLD: REPORTS FROM IN VITRO STUDIES}

The findings from in vitro studies demonstrated that metformin could reduce lipid accumulation ${ }^{13}$ and de novo fatty acid synthesis. ${ }^{14-16}$ Several proteins have been shown to be essential to the regulation of hepatic de novo lipogenesis. For example, the enzyme acetyl-CoA carboxylase (ACC) catalyzes acetyl CoA into malonyl $\mathrm{CoA}$, a precursor for fatty acid hepatic synthesis, ACC playing a vital role in a rate limiting step of lipogenesis. ${ }^{17}$ Phosphorylation of ACC via AMP-activated protein kinase (AMPK) inhibits the action of ACC, leading to inhibition of lipogenesis. ${ }^{18,19}$ Metformin increased inhibitory phosphorylation of $\mathrm{ACC}^{13}$ and induced hepatic Rho-kinase 1 (ROCK1) inhibition, ${ }^{16}$ resulting in AMPK activation and a decrease in lipogenic genes associated with de novo lipogenesis. ${ }^{16}$ Autophagy restoration via the sirtuin 1 (SIRT1) dependent pathway ${ }^{13}$ and signal transducer and activation of transcription 3 (STAT3) inhibition ${ }^{20}$ by metformin has been demonstrated. Anti-apoptotic activity, ${ }^{21}$ protection against lipidinduced necrotic cell death, ${ }^{21}$ reduction of oxidative stress ${ }^{21}$ and inflammatory markers ${ }^{20}$ were also shown in metformin treated cells. These in vitro reports are summarized in Supplementary Table 1.

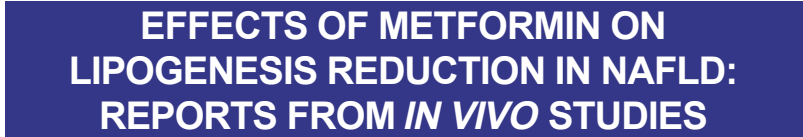

Several in vivo studies evaluated the effects of metformin on the reduction of hepatic fat content and the mechanism responsible. A variety of studies involving a range of dosages, routes, and durations of metformin treatment in genetically modified mice which exhibit features of hepatic steatosis, or dietary models of NAFLD rats or mice have been performed. Most of the studies demonstrate the effectiveness of intrahepatic lipid reduction by metformin..$^{13,14,16,20,22-28}$ However, there are a few contradictory reports showing ineffectiveness of metformin treatment. ${ }^{15,29-31}$ The differences in the NAFLD models used causing the varying degrees of disease severity, and accompanying metabolic derangement could be responsible for the discrepancies. It is observable that among the studies showing negative effects, the models with more severe disease were used, including the use of mice feeding with higher percentage of fat in high fat diet (HFD), ${ }^{15}$ methionine- and choline-deficient diet, ${ }^{29}$ Zucker diabetic fatty rat, ${ }^{30}$ and Goto-Kakizaki rat fed with HFD. ${ }^{31}$ The dosing and route of metformin administration were also varied between studies, and this could potentially affect the drug absorption with all studies using intraperitoneal route administration showing positive effects. . $^{13,14,20}$

\section{Effects of metformin on molecular mechanisms of hepatic steatosis (de novo lipogenesis reduction and increased fatty acid $\beta$-oxidation)}

Metformin is known to activate AMPK. ${ }^{32}$ The inhibition of phosphorylation of ACC by AMPK resulting in de novo lipogenesis reduction is one of the most widely mentioned responsible mechanisms. ${ }^{14,16,22,24,30}$ An ACC knockin mouse model had increased liver triglyceride (TG) content and increased liver fibrosis. ${ }^{14}$ Metformin treatment decreased hepatic lipogenesis and liver TG content in wildtype mice but not in ACC knock-in mice. These findings suggested that inhibition of phosphorylation of ACC by AMPK was essential in metformin action. ${ }^{14}$ Other studies added weight to this by demonstrating increasing AMPK activation and decreasing hepatic TG content in mice treated with metformin. ${ }^{16,22,24,30}$ It has been proposed that AMPK activation was mediated by ROCK $1 .{ }^{16}$ Lipogenic gene expression of proteins involved in hepatic lipogenesis, including sterol regulatory element-binding protein 1 (SREBP-1c), ${ }^{16,23}$ ACC, ${ }^{23}$ fatty acid synthase (FAS) ${ }^{16,23}$ and stearoyl-CoA desaturase-1 (SCD1) ${ }^{16}$ were reduced with metformin treatment. It was speculated that these changes were related to the activation of AMPK. ${ }^{16,33}$ 
Leptin is an adipose tissue-produced peptide which decreases hepatic de novo fatty acid synthesis and promotes peroxisome proliferator-activated receptor gamma coactivator- $1 \alpha(\operatorname{PPAR} \alpha)$-dependent fatty acid beta oxidation. ${ }^{34}$ Circulating leptin levels were found to be higher in NAFLD patients than controls ${ }^{35}$ and it was proposed that the blunted response of the liver to leptin action was related to hepatic steatosis. ${ }^{36}$ An enhanced leptin sensitivity by metformin is one of potential mechanisms underlying its steatosis alleviation effect. ${ }^{23}$ However, a study in Zucker diabetic fatty rats, those with missense mutation in the leptin receptor gene which develop early fatty liver, severe hyperlipidemia, and insulin resistance, ${ }^{37}$ showed that metformin had no effect on NAFLD. ${ }^{30}$ This finding may imply the leptin gene is essential for metformin treatment to be effective, or suggest that the extent of the effect of metformin was not enough in the case of a more severe and early onset of disease. Proteins involved in mitochondrial lipid oxidation were up-regulated following metformin treatment ${ }^{38}$ suggesting the potential effect of metformin in increasing mitochondrial lipid oxidation. All these reports are summarized in Table 1.

\section{Effects of metformin on hepatic inflammation, oxidative stress, and fibrosis}

Tumor necrosis factor- $\alpha$ (TNF- $\alpha$ ) is known to be a mediator of apoptosis and hepatotoxicity. ${ }^{39}$ It is also involved in NAFLD development and NASH progression. ${ }^{40}$ The results regarding TNF- $\alpha$ level upon metformin treatment are inconsistent, showing both reduction in ${ }^{20,25,26,31}$ and neutral TNF- $\alpha$ levels. ${ }^{28,29}$ Other inflammatory markers, including, inducible nitric oxide synthase (iNOS), ${ }^{25,26}$ interleukin$1 \beta,{ }^{20}$ transforming growth factor $\beta$ (TGF- $\beta$ ), ${ }^{28}$ and CD68 ${ }^{22}$ decreased upon metformin treatment. While the markers of inflammation decreased, the oxidative stress parameters glutathione and superoxide dismutase (SOD), ${ }^{25}$ and the antioxidant protein peroxiredoxin 6 (PRDX-6) ${ }^{38}$ increased after metformin treatment. These findings provide a potential mechanism for metformin in treatment of NAFLD by alleviating inflammation in the liver and decreasing oxidative stress. All these reports are summarized in Table 2.

\section{Direct degradation of intracellular lipid by autophagy induction}

The induction of autophagy enables cells to reutilize their own constituents for energy, one of the approaches for NAFLD treatment. ${ }^{41}$ The downregulation of SIRT1 expression and autophagy induction in the liver of $o b / o b$ mice were restored following treatment with metformin. ${ }^{13}$ Additionally, metformin was shown to inhibit the STAT3 pathway, ${ }^{20}$ the pathway in which inhibition also induced autophagy. ${ }^{42}$ All these reports are summarized in Table 3.

\section{Other proposed mechanisms of metformin}

Apolipoprotein A-I (ApoA-I) may be involved in the treatment effects of metformin as its deficiency increased mice sensitivity to diet-induced obesity ${ }^{43}$ and blunted the beneficial effect of metformin on liver lipid content. ${ }^{24}$ Metformin alters the enzymes and genes associated with NAFLD development. Deficiency of the enzyme glycine $\mathrm{N}$-methyltransferase (GNMT) which has a crucial role in NAFLD development, ${ }^{44}$ was up-regulated upon treatment. ${ }^{38}$ It also induced transcriptome alteration which is negatively correlated with liver disease and injuries, ${ }^{22}$ and induced changes in gene expression associated with the NAFLD phenotypes. ${ }^{22}$

Intestinal dysbiosis and gut barrier function were found to be associated with the development of NAFLD. ${ }^{45}$ The mechanism behind the protective effects of metformin against NAFLD development could be partly due to the modulation of the population of intestinal microbiota, ${ }^{27}$ protection against tight junction protein loss, ${ }^{26}$ and the reduction of bacterial endotoxins. ${ }^{26,27}$ All these reports are summarized in Supplementary Table 2. A summary of in vivo and in vitro reports, regarding the mechanisms behind the action of metformin and lipogenesis reduction in the NAFLD model is also shown in Fig. 1.

\section{EFFECTS OF METFORMIN ON THE LIVER IN NAFLD PATIENTS WITH DIABETES}

Preclinical studies showed remarkable improvement in liver histology and in the reduction of hepatic fat content following treatment with metformin as mentioned earlier. Therefore, metformin was expected to be a promising medication against NASH. However, metformin had limited impact in clinical studies among NAFLD or NASH patients without diabetes. ${ }^{7,8,12}$ The question remained to be answered is whether metformin treatment in NAFLD patients with diabetes could provide any clinical benefit since it is accepted as a safe medication and is widely used. Here we summarized available clinical reports on NAFLD patients with diabetes, including the effects of metformin as a monotherapy, comparison of metformin to other antidiabetic medications and as part of a combination treatment.

\section{Effects of metformin as a monotherapy in NAFLD patients with diabetes}

The efficacy of metformin monotherapy in the NAFLD population with diabetes has rarely been evaluated in a randomized-controlled study. The majority of the clinical 


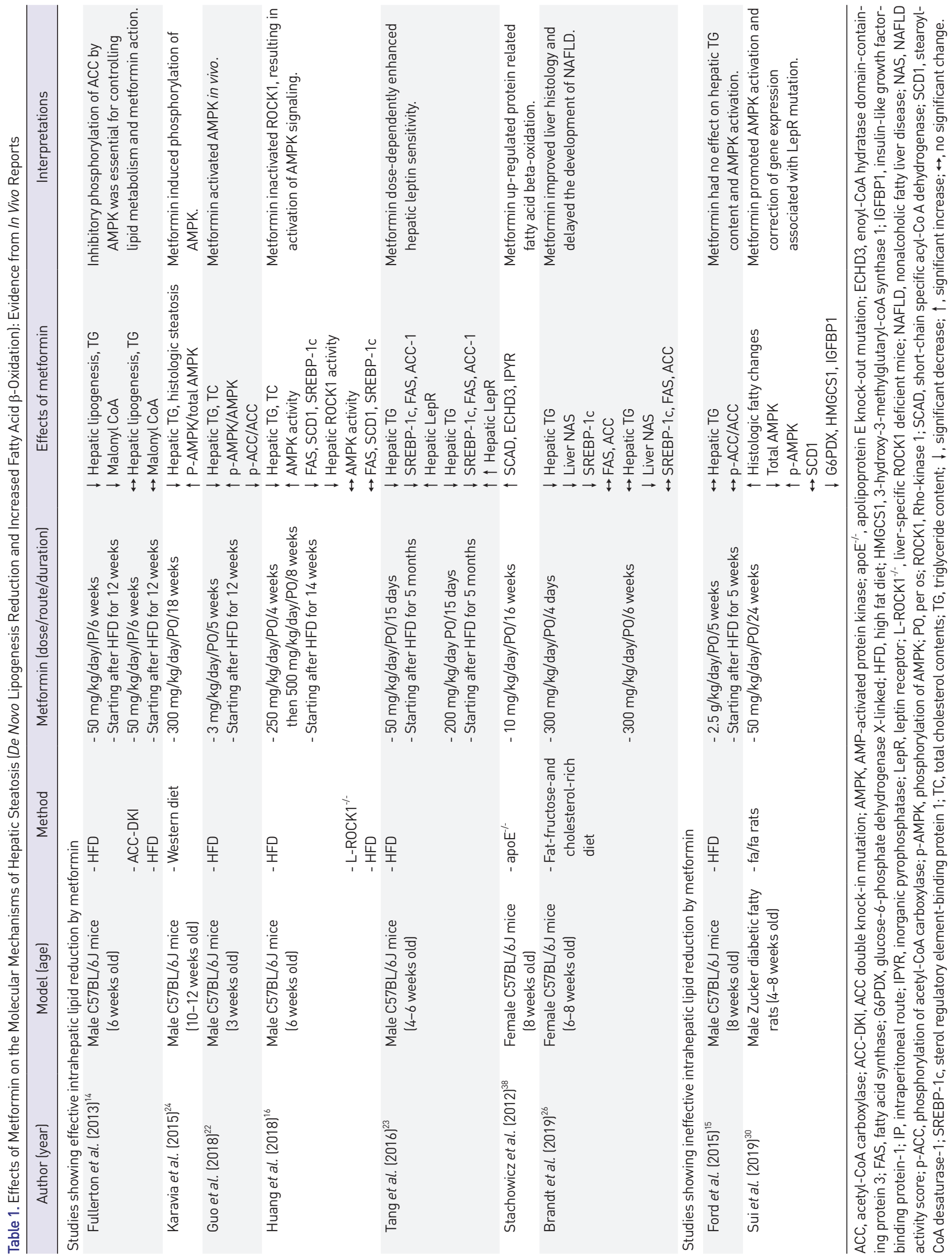




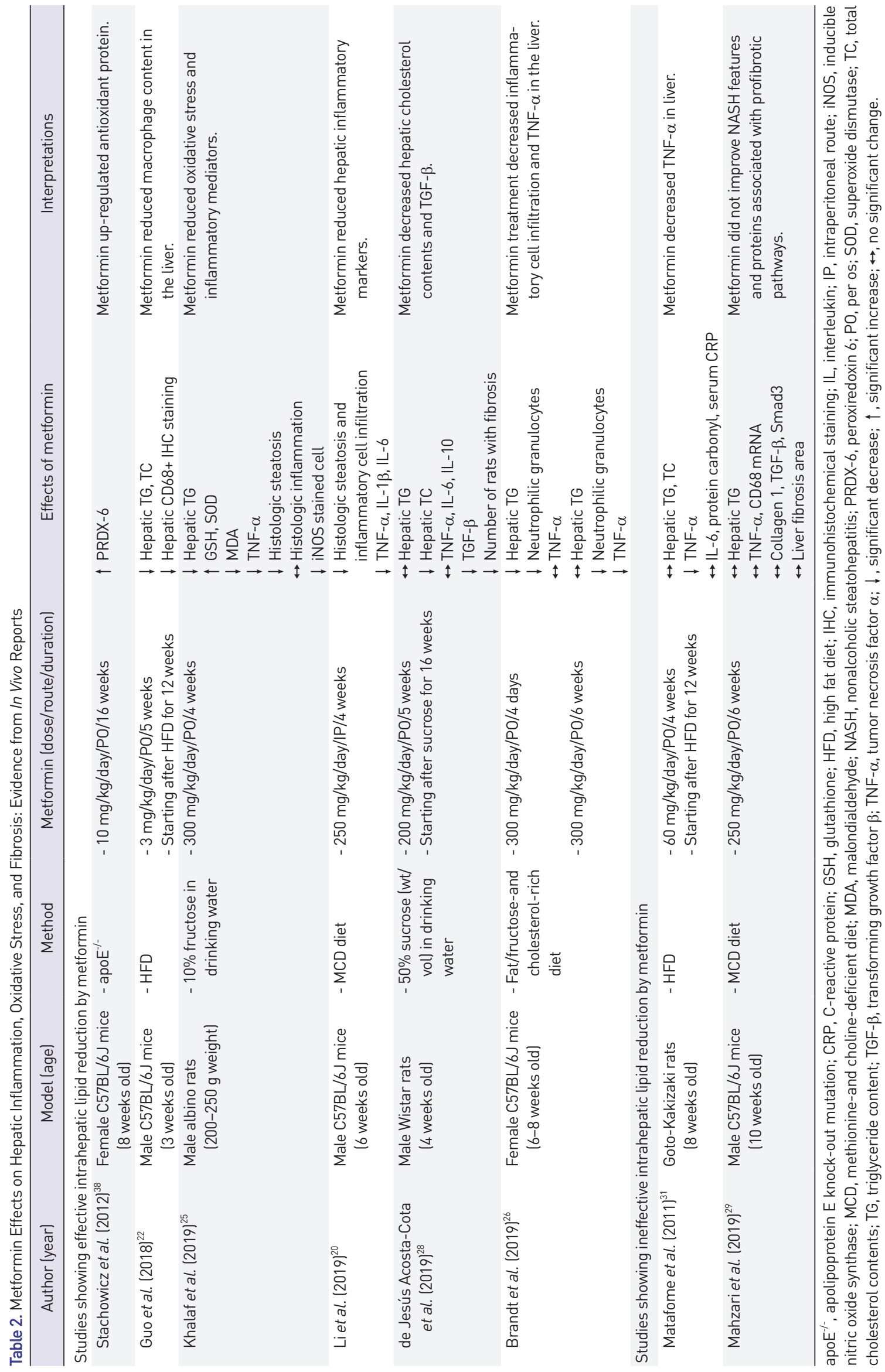




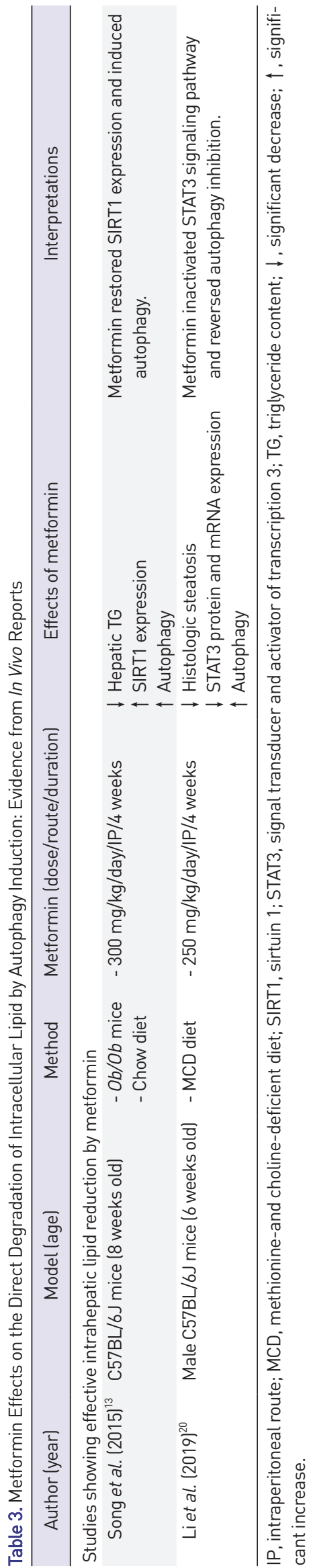

studies were conducted with the primary aim of comparing the effects of metformin with other antidiabetic medications. No placebo-controlled study has been conducted at this time. In eight studies that reported the effects of metformin in diabetic NAFLD patients compared to the pretreatment baseline condition, the patients were treated with metformin at dosages ranging from 1,000 to 2,000 $\mathrm{mg}$ /day for 12 to 48 weeks. ${ }^{46-53}$ These studies had various methods of NAFLD diagnosis, including ultrasonographic assessment of hepatic steatosis, a quantitative ultrasonographic method, or a liver/spleen computed tomography ratio (L/S CT ratio) of less than 1 . One study included the patients who underwent liver biopsy and were diagnosed as $\mathrm{NASH}^{51}$

Metformin was shown to be beneficial in patients with NAFLD compared to baseline. Five studies showed that metformin treatment for 12 to 24 weeks reduced the body mass index (BMI), liver fat content, liver enzymes, and hemoglobin $\mathrm{A} 1 \mathrm{c}(\mathrm{HbA1c})$ and improved insulin resistance in NAFLD patients with type 2 diabetes mellitus (T2DM). ${ }^{46-50}$ A prospective study in 11 patients with new-onset T2DM showed lower amounts of fat in the liver after 16 weeks of metformin treatment. ${ }^{53}$ However, one study reported inconsistent findings. In this small study with 16 participants treated with metformin for 24 weeks, increased liver fat content was demonstrated, and no beneficial effects of metformin were found on the BMI, transaminase level, and HbAlc. ${ }^{52}$ The possible cause of this conflicting result could be due partly to the limited number and type of patients enrolled. In this study, the enrolled patients were older than other studies (mean age of 60 years) with slightly lower baseline HbA1c compared to other studies (mean HbA1c of 7.4\%). These patients' characteristics suggest longer NAFLD disease duration and less insulin resistance in the enrolled patients. Future studies are needed to test this hypothesis.

Metformin treatment significantly decreased liver fibrosis evaluated by noninvasive measurement in NAFLD patients. ${ }^{46}$ However, inconsistent report exists. No significant improvement of fibrosis was demonstrated in histologic NASH patients $(n=10)$ evaluated by liver biopsy. ${ }^{51}$ The number of patients included in this study was small, which could limit the study power. Since this study also enrolled both T2DM and impaired glucose tolerance patients, the mean $\mathrm{HbAlc}$ at baseline (5.8\%) was lower than other studies. Several studies already showed that metformin was ineffective among patients without diabetes, ${ }^{7,812}$ thus further investigations in diabetes population are required. All these reports are summarized in Table 4.

Similar to findings from preclinical studies, metformin use is associated with decreased liver fat content in diabetic 


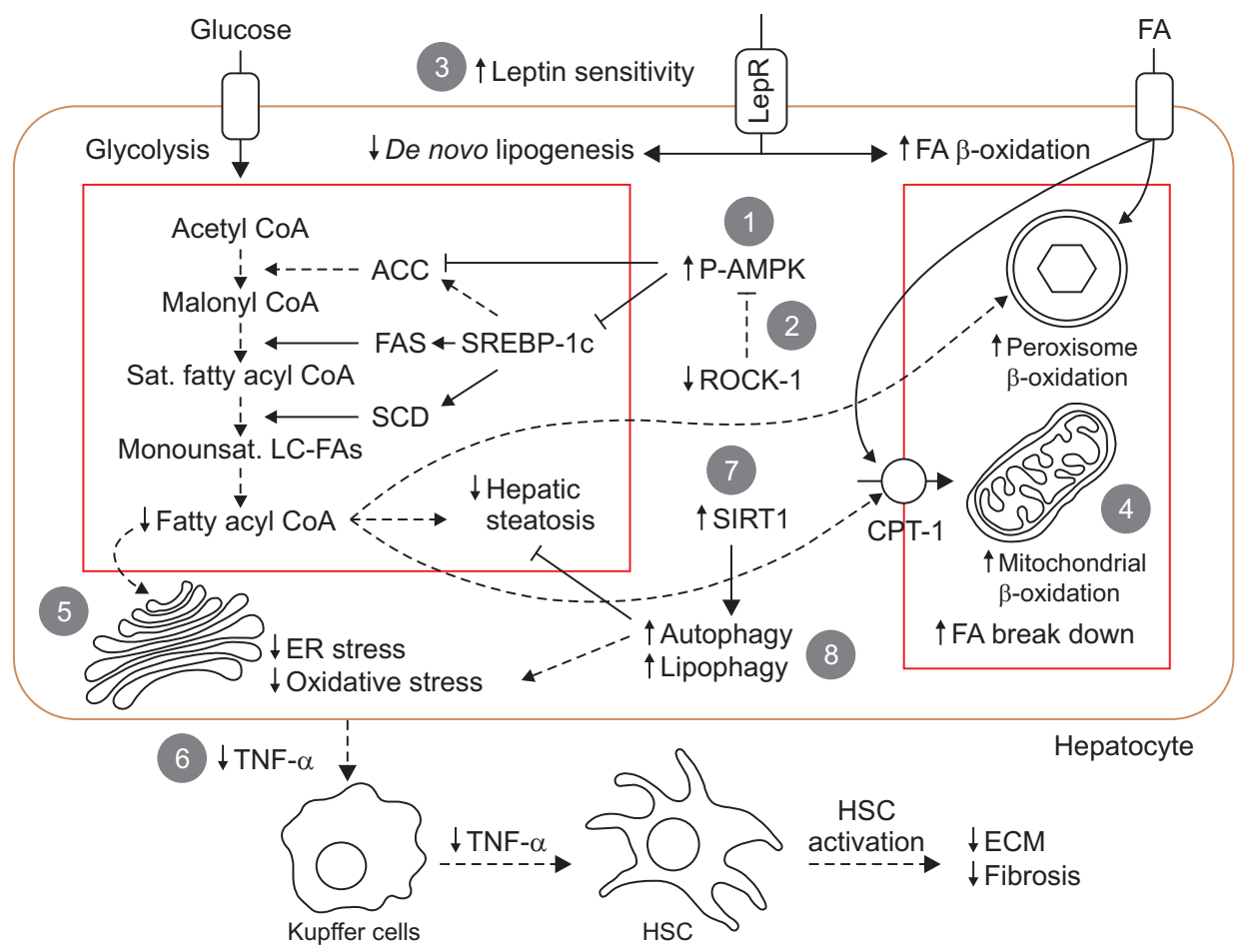

Fig. 1. Mechanism of action of metformin in nonalcoholic fatty liver disease. (A) Decrease in de novo lipogenesis: (1) AMPK activation and increase inhibitory phosphorylation of ACC; (2) inhibition of ROCK-1 by metformin resulting in inhibitory phosphorylation of ACC; and (3) increase in leptin sensitivity attenuates de novo lipogenesis pathway. Decreasing fatty acyl CoA also decreases hepatic steatosis, decreases lipid-induced ER stress and decreases substrate for FA $\beta$-oxidation. (B) Increase in FA $\beta$-oxidation: (3) increase in leptin sensitivity induces PPAR $\alpha$-dependent FA $\beta$-oxidation; (4) up-regulation of proteins involved in mitochondrial lipid oxidation by metformin results in increased FA breakdown and energy combustion. (C) Decrease in inflammation and HSC activation: (5) decreased lipid-induced ER stress and oxidative stress due to decreased de novo lipogenesis; (6) TNF- $\alpha$ reduction decreases Kupffer cell and HSC activation resulting in reducing inflammation and fibrosis in the liver. (D) Direct degradation of intracellular lipid: $(7,8)$ induction of autophagy by restoration of SIRT1 activity causing lipolysis by lysosome (lipophagy).

ACC, acetyl-CoA carboxylase; CPT-1, carnitine palmitoyltransferase; ECM, extracellular matrix proteins; ER, endoplasmic reticulum; FA, fatty acid; FAS, fatty acid synthase; HSC, hepatic stellate cells; LepR, leptin receptor; Monounsat. LC-FAs, monounsaturated long-chain FAs; P-AMPK, phosphorylated AMP-activated protein kinase; PPAR $\alpha$, peroxisome proliferator-activated receptor gamma coactivator-1 $\alpha$; ROCK1, Rho-kinase 1; Sat., saturated; SCD, stearoyl-CoA desaturase; SIRT1, sirtuin 1; SREBP-1c, sterol regulatory element-binding protein 1 ; TNF- $\alpha$, tumor necrosis factor.

patients. However, the effect is not as prominent as the effect shown in the rodent studies as the liver histology improvement was not replicated. It is unclear why there is a difference between animal and human studies. We speculate that this might be due to the uniform pattern of fatty phenotype in the animals studied, and the difference in the pharmacokinetics of metformin between species. Animal studies with an NAFLD model include genetically modified mice or mice fed with a diet promoting the development of a fatty liver. These models generate uniform fatty rodents and the effect of metformin might be seen more clearly than in a human study in which the participants had various degrees of severity of fatty liver and concurrent metabolic derangements. Metformin is taken up in the liver via organic cation transporter-1 (OCT1). ${ }^{54}$ Hepatic uptake of various drugs via this transporter has been shown to have differ between species, for example between mice and humans..$^{55}$ To our knowledge, no previous research had explored the species difference of metformin uptake. Therefore, it remains unclear whether our speculation would impact the results. Future research should examine this issue as it is important when projecting animal research results to humans. Histologic outcome should be further evaluated in a larger NAFLD population with diabetes. The effect of long-term treatment of metformin on liver-related adverse events are currently unclear, knowledge surrounding this is desirable.

Most of the recent studies conducted in diabetic NAFLD patients enrolled the patients treated with metformin and allowed metformin continuation during the study. Some studies included both metformin users and nonusers. However, despite modest effects being observed, metformin monotherapy decreased liver transaminases and hepatic fat content. These effects were prominent during the 12 to 24 weeks after administration. Therefore, the conduction of clinical studies should consider this possible effect for patient selection to avoid confounding effects caused by metformin treatment. 


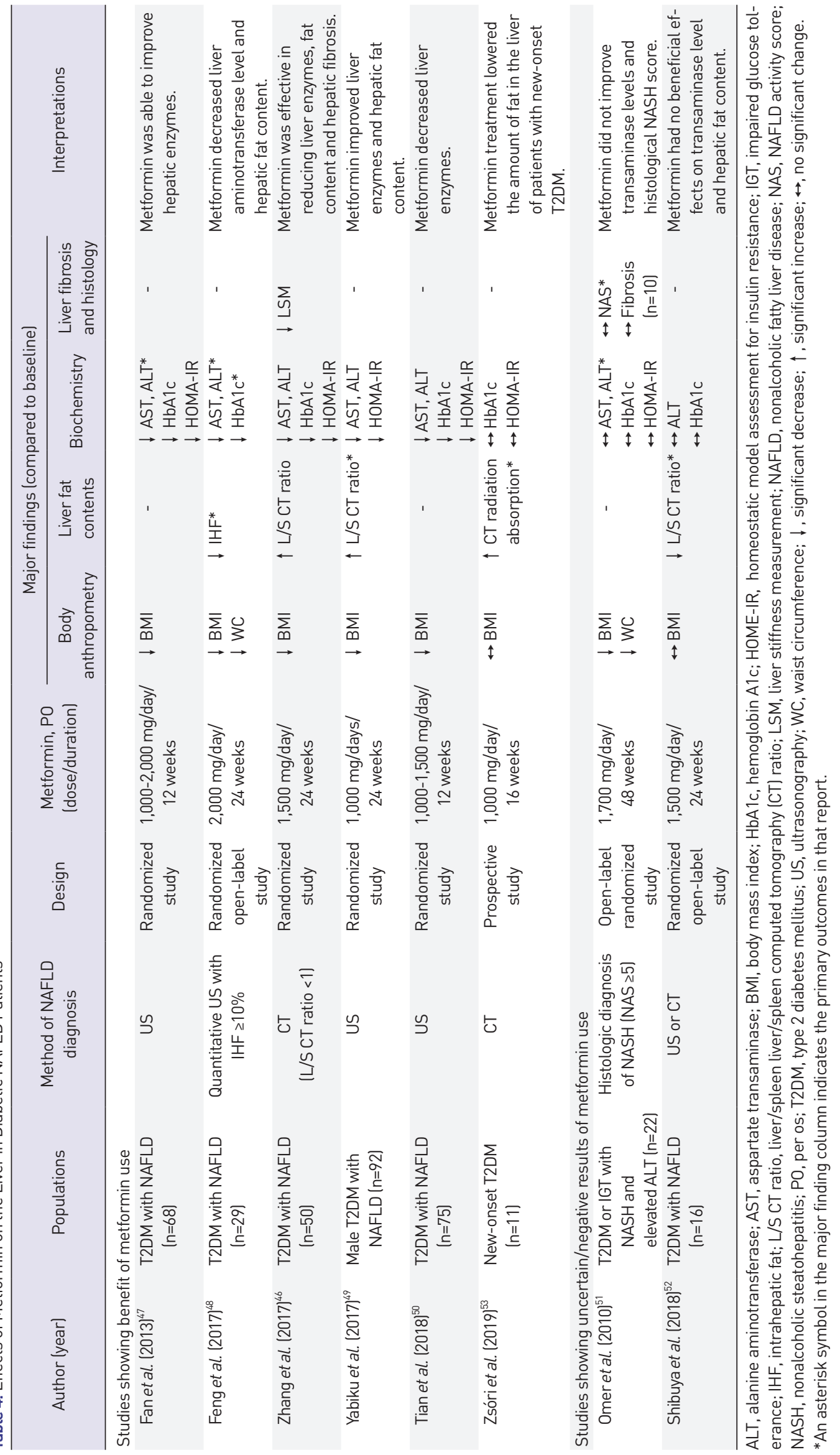




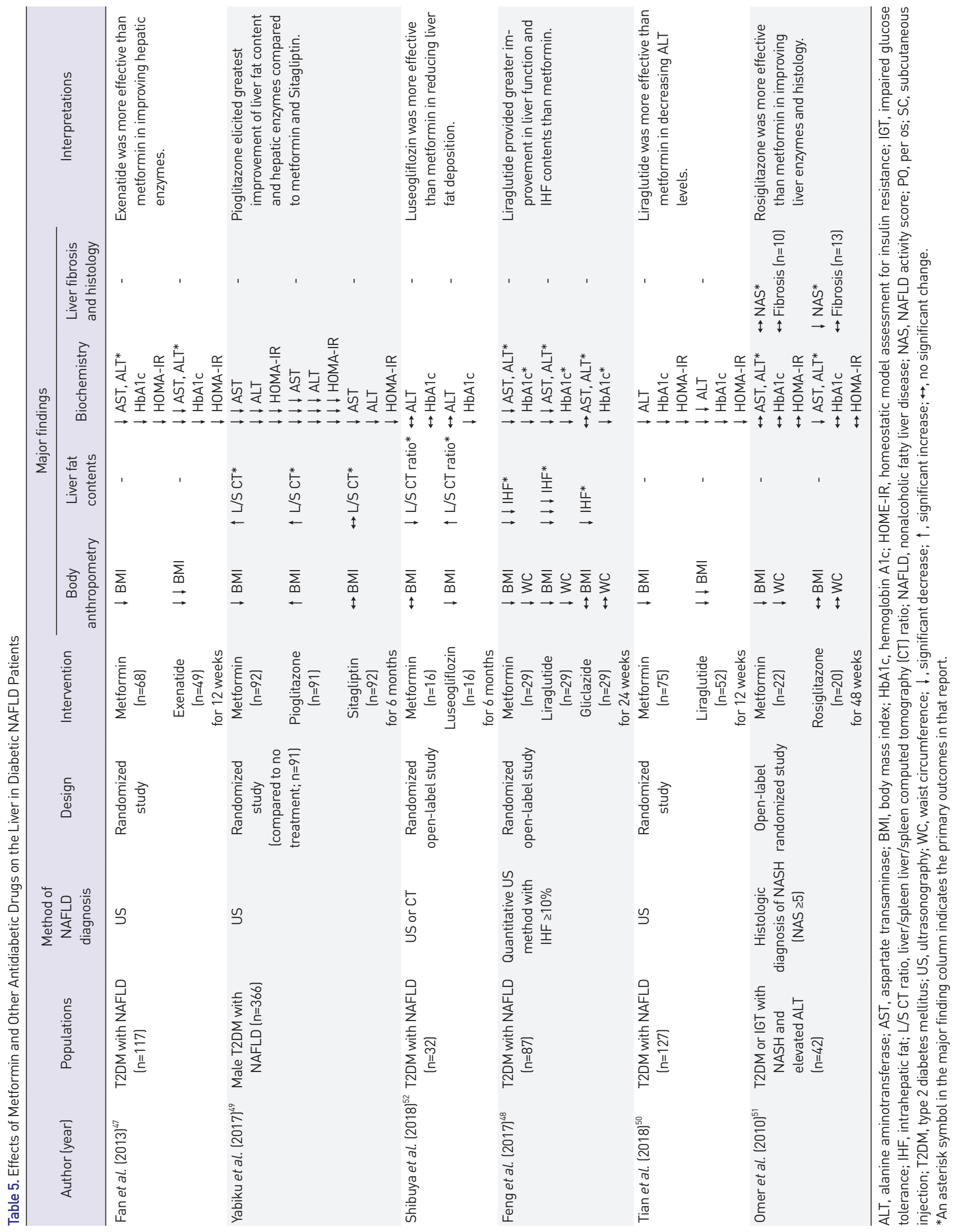




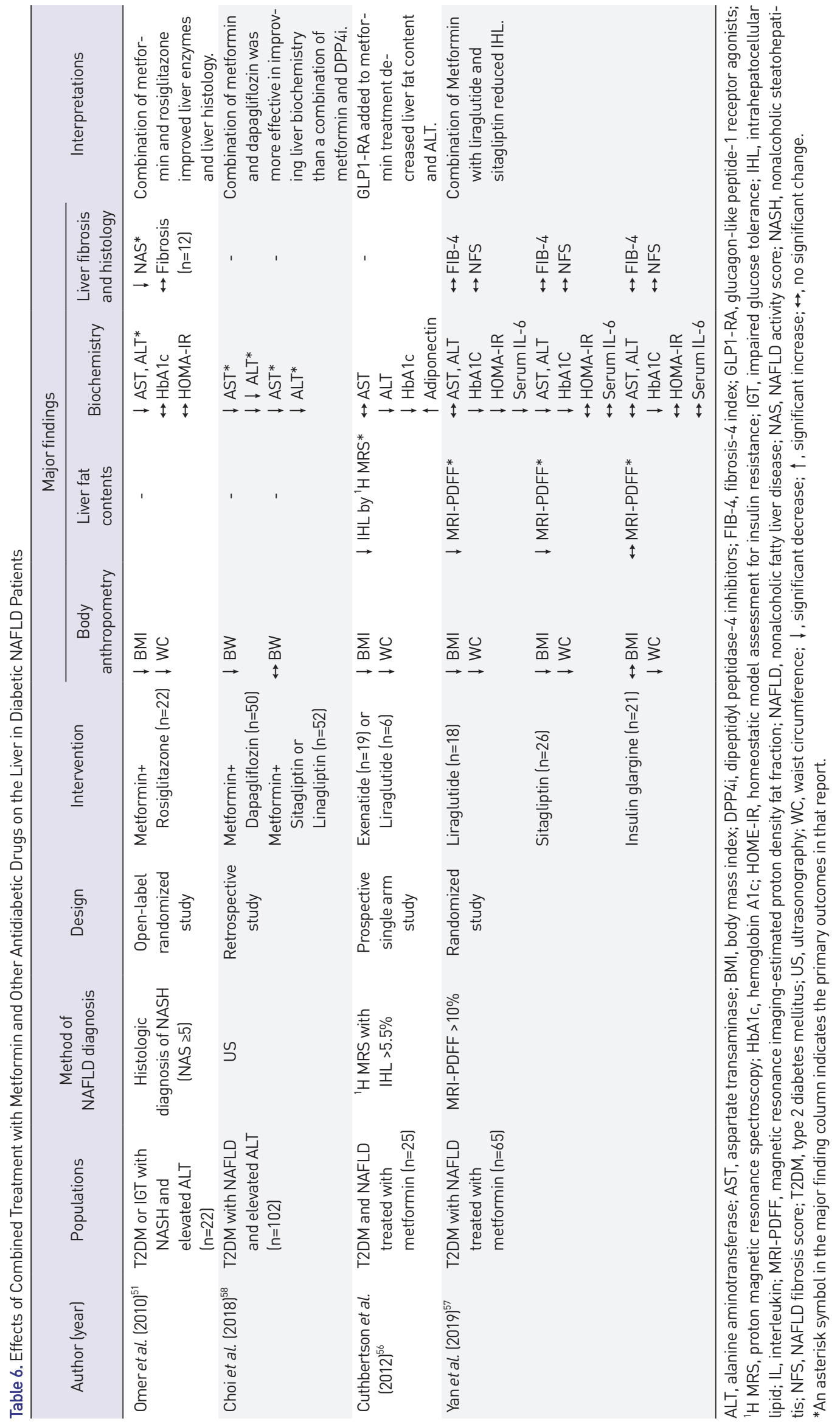




\section{Effects of metformin compared to other antidiabetic drugs in NAFLD patients with diabetes}

In the past decades, new classes of antidiabetic drugs have been approved to be used in T2DM patients. Although metformin as a monotherapy has been shown to reduce hepatic steatosis and improve liver biochemistry in diabetic NAFLD patients, the magnitude of the benefits seems to be more subtle than the newer antidiabetic drugs. These newer agents include thiazolidinediones, ${ }^{49,51}$ glucagon-like peptide-1 (GLP-1) receptor agonists, ${ }^{47,48,50}$ and sodium-glucose co-transporter-2 (SGLT2) inhibitors. ${ }^{52}$

Sitagliptin, a dipeptidyl peptidase-4 inhibitor (DPP4i), had less potency than metformin in reducing liver enzymes. ${ }^{49}$ Gliclazide, a drug in sulfonylurea class, was able to decrease hepatic fat content but to a lower extent than that observed after metformin therapy. ${ }^{48} \mathrm{~A}$ summary of reports comparing the effects of metformin to other antidiabetic drugs on the liver in diabetic NAFLD patients is shown in Table 5.

\section{Effects of combined treatment with metformin and other antidiabetic drugs in NAFLD patients with diabetes}

A combination of metformin with other antidiabetic drugs in NAFLD patients with diabetes had been studied and reported. Since most of diabetic patients had previously received metformin, enrollment of these patients with other antidiabetic medications being added on was common across most of these studies. Addition of thiazolidinediones, ${ }^{51}$ GLP-1 receptor agonists, ${ }^{56,57}$ DPP $4 \mathrm{i}^{57}$ and SGLT $2 \mathrm{i}^{58}$ all provided additional benefit to metformin as a monotherapy. However, it should be noted that the synergistic effect of metformin added on to other antidiabetic

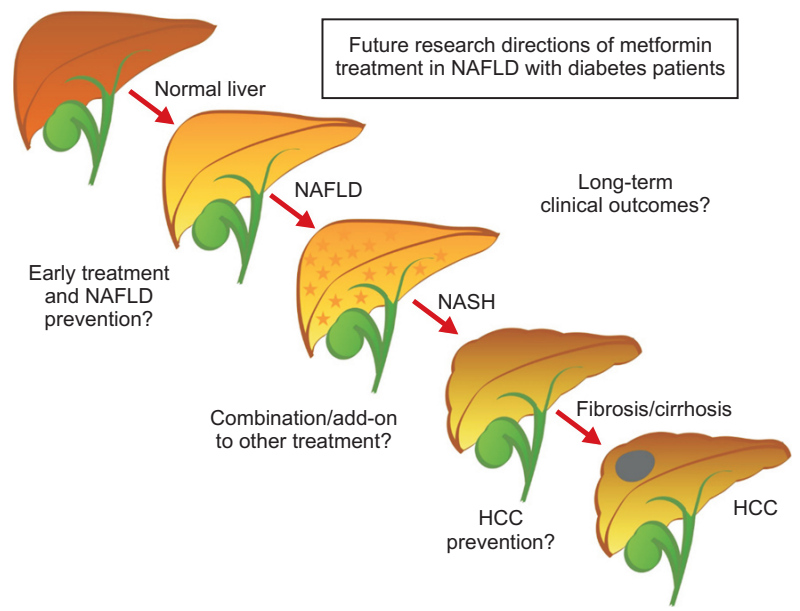

Fig. 2. Future directions.

NAFLD, nonalcoholic fatty liver disease; NASH, nonalcoholic steatohepatitis; HCC, hepatocellular carcinoma. medications has not yet been reported. Insulin glargine treatment did not improve NAFLD parameters further, as insulin treatment did not affect the insulin resistance nor body weight reduction. ${ }^{57}$ A summary of the reports regarding the effects of a combined treatment with metformin and other antidiabetic drugs on the liver in NAFLD patients with diabetes is shown in Table 6.

\section{EFFECTS OF METFORMIN ON HCC DEVELOPMENT}

The use of metformin was associated with a reduced risk of hepatocellular carcinoma (HCC).$^{59}$ Several epidemiological studies suggested that metformin had potential antitumor effect with potential effects in cancer prevention. $^{60,61}$ A large matched-paired cohort conducted in Taiwan found that metformin was associated with HCC incidence reduction in patients with $\mathrm{T} 2 \mathrm{DM}$ with a hazard ratio of $0.76(0.67$ to 0.85$) .{ }^{62}$ In a mouse model of NASH and liver tumor, metformin decreased the proportion of tumorcarrying mice. ${ }^{63}$ However, this effect was not observed in the liver in mice that had already developed NAFLD. ${ }^{63}$ Another study of a HFD-fed, HCC model of transgenic zebrafish demonstrated the HFD enhanced malignancyrelated histologic and morphologic features. ${ }^{64}$ Metformin treatment reduced liver size and reversed the diet-induced increase in steatosis, vessel formation, and inflammation and restored $\mathrm{T}$ cell infiltration. ${ }^{64}$ These results suggested potential benefits of metformin in the prevention of HFDinduced liver tumorigenesis and progression, especially if administered early prior to the onset of NAFLD. Further studies are needed to warrant this benefit of metformin as regards liver cancer.

\section{CONCLUSION AND FUTURE PERSPECTIVES}

Metformin treatment was shown to be effective in alleviating hepatic lipogenesis in animal models of NAFLD through various mechanisms. However, in clinical studies, metformin could modestly reduce the BMI, liver fat content, and liver enzymes in NAFLD patients with diabetes. Despite these reports on benefits of metformin, some contradicting reports still exist. Combination treatments with other antidiabetic drugs, especially the drugs in the thiazolidinediones, GLP-1 receptor agonists and SGLT2 inhibitors groups demonstrated increased efficacy. Among diabetic patients with biopsy-proven NASH, currently available data from a small enrolled study suggested that 
metformin was not associated with histologic or liver fibrosis improvement. Further research with a larger sample size is warranted to confirm these findings. A long-term clinical study to evaluate liver-related complications, and a study to elucidate the role of metformin in HCC prevention are necessary. Summaries of the future directions are shown in Fig. 2. Nevertheless, there is a potential benefit in the continued use of metformin in NAFLD patients with diabetes, either alone or in combination with other antidiabetic drugs.

\section{CONFLICTS OF INTEREST}

No potential conflict of interest relevant to this article was reported.

\section{ACKNOWLEDGEMENTS}

This work was supported by the NSTDA Research Chair grant from the National Science and Technology Development Agency Thailand (N.C.), and the Chiang Mai University Center of Excellence Award (N.C.).

\section{ORCID}

Kanokwan Pinyopornpanish https://orcid.org/0000-0002-5231-7582

Apinya Leerapun https://orcid.org/0000-0001-7951-3359 Kanokporn Pinyopornpanish https://orcid.org/0000-0001-9068-3834 Nipon Chattipakorn

https://orcid.org/0000-0003-3026-718X

\section{REFERENCES}

1. Allen AM, Therneau TM, Larson JJ, Coward A, Somers VK, Kamath PS. Nonalcoholic fatty liver disease incidence and impact on metabolic burden and death: a 20 year-community study. Hepatology 2018;67:1726-1736.

2. Sanyal AJ, Campbell-Sargent C, Mirshahi F, et al. Nonalcoholic steatohepatitis: association of insulin resistance and mitochondrial abnormalities. Gastroenterology 2001;120:11831192.

3. Williams CD, Stengel J, Asike MI, et al. Prevalence of nonalcoholic fatty liver disease and nonalcoholic steatohepatitis among a largely middle-aged population utilizing ultrasound and liver biopsy: a prospective study. Gastroenterology
2011;140:124-131.

4. Lomonaco R, Bril F, Portillo-Sanchez P, et al. Metabolic impact of nonalcoholic steatohepatitis in obese patients with type 2 diabetes. Diabetes Care 2016;39:632-638.

5. Rena G, Hardie DG, Pearson ER. The mechanisms of action of metformin. Diabetologia 2017;60:1577-1585.

6. Li Y, Liu L, Wang B, Wang J, Chen D. Metformin in nonalcoholic fatty liver disease: a systematic review and metaanalysis. Biomed Rep 2013;1:57-64.

7. Haukeland JW, Konopski Z, Eggesbø HB, et al. Metformin in patients with non-alcoholic fatty liver disease: a randomized, controlled trial. Scand J Gastroenterol 2009;44:853-860.

8. Uygun A, Kadayifci A, Isik AT, et al. Metformin in the treatment of patients with non-alcoholic steatohepatitis. Aliment Pharmacol Ther 2004;19:537-544.

9. Bugianesi E, Gentilcore E, Manini R, et al. A randomized controlled trial of metformin versus vitamin E or prescriptive diet in nonalcoholic fatty liver disease. Am J Gastroenterol 2005;100:1082-1090.

10. Duseja A, Das A, Dhiman RK, et al. Metformin is effective in achieving biochemical response in patients with nonalcoholic fatty liver disease (NAFLD) not responding to lifestyle interventions. Ann Hepatol 2007;6:222-226.

11. Nair S, Diehl AM, Wiseman M, Farr GH Jr, Perrillo RP. Metformin in the treatment of non-alcoholic steatohepatitis: a pilot open label trial. Aliment Pharmacol Ther 2004;20:2328.

12. Shields WW, Thompson KE, Grice GA, Harrison SA, Coyle WJ. The effect of metformin and standard therapy versus standard therapy alone in nondiabetic patients with insulin resistance and nonalcoholic ateatohepatitis (NASH): a pilot trial. Therap Adv Gastroenterol 2009;2:157-163.

13. Song YM, Lee YH, Kim JW, et al. Metformin alleviates hepatosteatosis by restoring SIRT1-mediated autophagy induction via an AMP-activated protein kinase-independent pathway. Autophagy 2015;11:46-59.

14. Fullerton MD, Galic S, Marcinko K, et al. Single phosphorylation sites in Acc1 and Acc2 regulate lipid homeostasis and the insulin-sensitizing effects of metformin. Nat Med 2013;19:1649-1654.

15. Ford RJ, Fullerton MD, Pinkosky SL, et al. Metformin and salicylate synergistically activate liver AMPK, inhibit lipogenesis and improve insulin sensitivity. Biochem J 2015;468:125-132.

16. Huang H, Lee SH, Sousa-Lima I, et al. Rho-kinase/AMPK axis regulates hepatic lipogenesis during overnutrition. J Clin Invest 2018;128:5335-5350.

17. Sanders FW, Griffin JL. De novo lipogenesis in the liver in health and disease: more than just a shunting yard for glucose. Biol Rev Camb Philos Soc 2016;91:452-468.

18. Brownsey RW, Boone AN, Elliott JE, Kulpa JE, Lee WM. 
Regulation of acetyl-CoA carboxylase. Biochem Soc Trans 2006;34(Pt 2):223-227.

19. Viollet B, Foretz M, Guigas B, et al. Activation of AMPactivated protein kinase in the liver: a new strategy for the management of metabolic hepatic disorders. J Physiol 2006;574(Pt 1):41-53.

20. Li YL, Li XQ, Wang YD, Shen C, Zhao CY. Metformin alleviates inflammatory response in non-alcoholic steatohepatitis by restraining signal transducer and activator of transcription 3-mediated autophagy inhibition in vitro and in vivo. Biochem Biophys Res Commun 2019;513:64-72.

21. Geng Y, Hernández Villanueva A, Oun A, et al. Protective effect of metformin against palmitate-induced hepatic cell death. Biochim Biophys Acta Mol Basis Dis 2020;1866:165621.

22. Guo J, Zhou Y, Cheng Y, et al. Metformin-induced changes of the coding transcriptome and non-coding RNAs in the livers of non-alcoholic fatty liver disease mice. Cell Physiol Biochem 2018;45:1487-1505.

23. Tang X, Li J, Xiang W, et al. Metformin increases hepatic leptin receptor and decreases steatosis in mice. J Endocrinol 2016;230:227-237.

24. Karavia EA, Hatziri A, Kalogeropoulou C, et al. Deficiency in apolipoprotein A-I ablates the pharmacological effects of metformin on plasma glucose homeostasis and hepatic lipid deposition. Eur J Pharmacol 2015;766:76-85.

25. Khalaf HM, Ibrahim MA, Amin EF, Abdel-Tawab Ibrahim S, Abdel-Wahab S, Fouad YM. Allopurinol potentiates the hepatoprotective effect of metformin and vitamin $\mathrm{E}$ in fructoseinduced fatty liver in rats. Clin Exp Hepatol 2019;5:65-74.

26. Brandt A, Hernández-Arriaga A, Kehm R, et al. Metformin attenuates the onset of non-alcoholic fatty liver disease and affects intestinal microbiota and barrier in small intestine. Sci Rep 2019;9:6668.

27. Shin NR, Bose S, Wang JH, et al. Flos Lonicera combined with metformin ameliorates hepatosteatosis and glucose intolerance in association with gut microbiota modulation. Front Microbiol 2017;8:2271.

28. de Jesús Acosta-Cota S, Aguilar-Medina EM, Ramos-Payán $\mathrm{R}$, et al. Therapeutic effect of treatment with metformin and/ or 4-hydroxychalcone in male Wistar rats with nonalcoholic fatty liver disease. Eur J Pharmacol 2019;863:172699.

29. Mahzari A, Li S, Zhou X, et al. Matrine protects against MCD-induced development of NASH via upregulating HSP72 and downregulating mTOR in a manner distinctive from metformin. Front Pharmacol 2019;10:405.

30. Sui Y, Kong X, Fan R, et al. Long-term treatment with metformin in the prevention of fatty liver in Zucker diabetic fatty rats. Diabetol Metab Syndr 2019;11:94.

31. Matafome P, Louro T, Rodrigues L, et al. Metformin and atorvastatin combination further protect the liver in type
2 diabetes with hyperlipidaemia. Diabetes Metab Res Rev 2011;27:54-62.

32. Hawley SA, Gadalla AE, Olsen GS, Hardie DG. The antidiabetic drug metformin activates the AMP-activated protein kinase cascade via an adenine nucleotide-independent mechanism. Diabetes 2002;51:2420-2425.

33. Li Y, Xu S, Mihaylova MM, et al. AMPK phosphorylates and inhibits SREBP activity to attenuate hepatic steatosis and atherosclerosis in diet-induced insulin-resistant mice. Cell Metab 2011;13:376-388.

34. Polyzos SA, Kountouras J, Mantzoros CS. Leptin in nonalcoholic fatty liver disease: a narrative review. Metabolism 2015;64:60-78.

35. Polyzos SA, Aronis KN, Kountouras J, Raptis DD, Vasiloglou MF, Mantzoros CS. Circulating leptin in non-alcoholic fatty liver disease: a systematic review and meta-analysis. Diabetologia 2016;59:30-43.

36. Fishman S, Muzumdar RH, Atzmon G, et al. Resistance to leptin action is the major determinant of hepatic triglyceride accumulation in vivo. FASEB J 2007;21:53-60.

37. Yokoi N, Hoshino M, Hidaka S, et al. A novel rat model of type 2 diabetes: the Zucker Fatty Diabetes Mellitus ZFDM rat. J Diabetes Res 2013;2013:103731.

38. Stachowicz A, Suski M, Olszanecki R, Madej J, Okoń K, Korbut R. Proteomic analysis of liver mitochondria of apolipoprotein E knockout mice treated with metformin. J Proteomics 2012;77:167-175.

39. Bradham CA, Plümpe J, Manns MP, Brenner DA, Trautwein C. Mechanisms of hepatic toxicity. I. TNF-induced liver injury. Am J Physiol 1998;275:G387-G392.

40. Kakino S, Ohki T, Nakayama H, et al. Pivotal role of TNF-a in the development and progression of nonalcoholic fatty liver disease in a murine model. Horm Metab Res 2018;50:8087.

41. Singh R. Autophagy and regulation of lipid metabolism. Results Probl Cell Differ 2010;52:35-46.

42. You L, Wang Z, Li H, et al. The role of STAT3 in autophagy. Autophagy 2015;11:729-739.

43. Karavia EA, Papachristou DJ, Liopeta K, Triantaphyllidou IE, Dimitrakopoulos O, Kypreos KE. Apolipoprotein A-I modulates processes associated with diet-induced nonalcoholic fatty liver disease in mice. Mol Med 2012;18:901-912.

44. Martínez-Chantar ML, Vázquez-Chantada M, Ariz U, et al. Loss of the glycine $\mathrm{N}$-methyltransferase gene leads to steatosis and hepatocellular carcinoma in mice. Hepatology 2008;47:1191-1199.

45. Bäckhed F, Ding H, Wang T, et al. The gut microbiota as an environmental factor that regulates fat storage. Proc Natl Acad Sci U S A 2004;101:15718-15723.

46. Zhang R, Cheng K, Xu S, et al. Metformin and diammonium glycyrrhizinate enteric-coated capsule versus metformin 
alone versus diammonium glycyrrhizinate enteric-coated capsule alone in patients with nonalcoholic fatty liver disease and type 2 diabetes mellitus. Gastroenterol Res Pract 2017;2017:8491742.

47. Fan H, Pan Q, Xu Y, Yang X. Exenatide improves type 2 diabetes concomitant with non-alcoholic fatty liver disease. Arq Bras Endocrinol Metabol 2013;57:702-708.

48. Feng W, Gao C, Bi Y, et al. Randomized trial comparing the effects of gliclazide, liraglutide, and metformin on diabetes with non-alcoholic fatty liver disease. J Diabetes 2017;9:800809.

49. Yabiku K, Mutoh A, Miyagi K, Takasu N. Effects of oral antidiabetic drugs on changes in the liver-to-spleen ratio on computed tomography and inflammatory biomarkers in patients with type 2 diabetes and nonalcoholic fatty liver disease. Clin Ther 2017;39:558-566.

50. Tian F, Zheng Z, Zhang D, He S, Shen J. Efficacy of liraglutide in treating type 2 diabetes mellitus complicated with non-alcoholic fatty liver disease. Biosci Rep 2018;38:BSR20181304.

51. Omer Z, Cetinkalp S, Akyildiz M, et al. Efficacy of insulinsensitizing agents in nonalcoholic fatty liver disease. Eur J Gastroenterol Hepatol 2010;22:18-23.

52. Shibuya T, Fushimi N, Kawai M, et al. Luseogliflozin improves liver fat deposition compared to metformin in type 2 diabetes patients with non-alcoholic fatty liver disease: a prospective randomized controlled pilot study. Diabetes Obes Metab 2018;20:438-442.

53. Zsóri G, Illés D, Ivány E, et al. In new-onset diabetes mellitus, metformin reduces fat accumulation in the liver, but not in the pancreas or pericardium. Metab Syndr Relat Disord 2019;17:289-295.

54. Wang DS, Jonker JW, Kato Y, Kusuhara H, Schinkel AH, Sugiyama Y. Involvement of organic cation transporter 1 in hepatic and intestinal distribution of metformin. J Pharmacol Exp Ther 2002;302:510-515.

55. Morse BL, Kolur A, Hudson LR, et al. Pharmacokinetics of
Organic Cation Transporter 1 (OCT1) substrates in Oct1/2 knockout mice and species difference in hepatic OCT1mediated uptake. Drug Metab Dispos 2020;48:93-105.

56. Cuthbertson DJ, Irwin A, Gardner CJ, et al. Improved glycaemia correlates with liver fat reduction in obese, type 2 diabetes, patients given glucagon-like peptide-1 (GLP-1) receptor agonists. PLoS One 2012;7:e50117.

57. Yan J, Yao B, Kuang H, et al. Liraglutide, sitagliptin, and insulin glargine added to metformin: the effect on body weight and intrahepatic lipid in patients with type 2 diabetes mellitus and nonalcoholic fatty liver disease. Hepatology 2019;69:2414-2426.

58. Choi DH, Jung CH, Mok JO, Kim CH, Kang SK, Kim BY. Effect of dapagliflozin on alanine aminotransferase improvement in type 2 diabetes mellitus with non-alcoholic fatty liver disease. Endocrinol Metab (Seoul) 2018;33:387-394.

59. Singh S, Singh PP, Singh AG, Murad MH, Sanchez W. Antidiabetic medications and the risk of hepatocellular cancer: a systematic review and meta-analysis. Am J Gastroenterol 2013;108:881-891.

60. Noto H, Goto A, Tsujimoto T, Noda M. Cancer risk in diabetic patients treated with metformin: a systematic review and meta-analysis. PLoS One 2012;7:e33411.

61. Kim HJ, Lee S, Chun $\mathrm{KH}$, et al. Metformin reduces the risk of cancer in patients with type 2 diabetes: an analysis based on the Korean National Diabetes Program Cohort. Medicine (Baltimore) 2018;97:e0036.

62. Tseng $\mathrm{CH}$. Metformin and risk of hepatocellular carcinoma in patients with type 2 diabetes. Liver Int 2018;38:2018-2027.

63. Tajima K, Nakamura A, Shirakawa J, et al. Metformin prevents liver tumorigenesis induced by high-fat diet in C57Bl/6 mice. Am J Physiol Endocrinol Metab 2013;305:E987-E998.

64. de Oliveira S, Houseright RA, Graves AL, et al. Metformin modulates innate immune-mediated inflammation and early progression of NAFLD-associated hepatocellular carcinoma in zebrafish. J Hepatol 2019;70:710-721. 